\title{
Studies on the Construction of Integrated Display Platform about Three-Dimensional Geological Mapping Model
}

\author{
Xiang LI and Mei Zhu \\ Software College of East China Institute of Technology \\ Nanchang, Jiangxi, China \\ *Corresponding author
}

\begin{abstract}
Three-dimensional geological survey was based on the surface geological mapping, which was to solve the major geological problems; it was a comprehensive three-dimensional geological survey, which used modern geological theories, advanced integrated exploration methods and three-dimensional visual information technology. The integrated comprehensive display on 3D geological model is the major means to solve the more types and complex sources of 3D geological mapping model. This paper combined actual application achievements of 3D geological model integrated display by Xiang shan's 3D geological survey, it also discussed and analyzed systematically the design ideology, system architecture and ideas of technical realization, in order to provide a new ideas and methods for domestic integrated display about three-dimensional geological model.
\end{abstract}

Keywords-integrated display platform; $3 D$ geological model; construction technology; universal 3D file interfaces

\section{INTRODUCTION}

Data visualization technology is the great processing ability with the computer system, the basic algorithm with graphic image and the visualizable algorithm to convert the huge amount of data for the static graphics, dynamic graphics or images, and presents in front of people, so that the hidden in the data without visible phenomenon become visible, for people to analyze, understand the data, concept formation and find out the rule it provides a powerful means. The data visualization technology at present has been widely used in social production, social life and so on, become being important significance on the national science-technology and national economy technology.

Three dimensional geological mapping is based on surface geological mapping, application of various geophysical prospecting, drilling and other exploration technology, obtaining geological information and research on crust surface a certain depth according to the corresponding precision (usually within $5 \mathrm{~km}$ ), to study the relationship between surface and underground geological body, 3D spatial geological map compilation. With the arrival of the era of 3D geological mapping, geological research achievements will produce huge amounts of data and very valuable. Because the information of geological bodies and phenomena is a function of three-dimensional space, and remote sensing, geological and geophysical information often has multi solutions, in the current geological application mode, geological research results are often limited to a platform presentation difference is big, easy to cause the understanding and application of the difficulties.

In order to make the results more intuitive visualization, easy to be understood and accepted experts and professionals in different fields, the construction of a based on does not depend on a specific visual platform open geological achievements visible application service platform, using the evaluation, and industry related prediction model, for extracting thematic information display is more important content of 3D geological mapping results socialization application. In this paper, combined with the integration of 3D geological survey of Xiangshan 3D model presentation applications, display integration design idea, system structure and technology platform for 3D geological mapping model, discusses the way and analysis system, with a view to display provides a new idea and method for the domestic three-dimensional geological model integration.

\section{GENERAL 3D SPECIFICATION}

Three general 3D specification (Universal 3D, referred to as U3D) is a shared virtual 3D graphics technology, it is a working group composed of Intel company and other companies, the 3D visualization technology and standard for a lightweight 3D industry forum to develop and launch the. It defines the syntax and semantics of Universal 3D files, one for the underlying 3D CAD data reuse and the visualization of the extensible format, all have effects on many mainstream business applications. Including the characteristics of this file format is obvious: runtime geometry changes, continuous level of detail, the compression of the specified range, the forward data flow and playback, key frames and the skeleton based dynamic, and the expansion and operation of the U3D format.

At the same time, the versatility of U3D's best features are still in it, which is not limited to software / hardware specific resources, independent of the special platform, so that shared 3D data easier cross platform, like in the audio MP3 and static image in JPEG, the U3D specification for 3D data extraction data encoding for a free access to, and can be widely used in the. PDF is one of the most important markets for U3D applications. Therefore, further study of U3D specification will lead to the specification further promotion in China, and to provide a reference for the general $3 \mathrm{D}$ data processing or storage mode of 
designers. At the same time, PDF is a file format developed by Adobe Company, more than one billion user group in the world. The PDF file is also very obvious advantages, such as low cost, easy to navigate, and platform independence, portability features good etc.. The combination provides interactive interface and realize the good technical support platform for the development of a system to show cross platform.

\section{DEMAND ANALYSIS}

Three dimensional geological mapping results of model integration platform to display the main goal is to combine the comprehensive expression results of 3D geological mapping project requirements, the fusion of geographic features, exploration of mine, geophysical information in many aspects, the realization of geological model Micromine, Mapgis, Surpac and many other professional software processing correctly show, and have a simple and appropriate man-machine interactive function. The platform should have the following characteristics:

1) Analysis of PDF model in U3D format and display on the screen. This is the most basic functions, the obtained model can be way has two kinds, one is through the file mode, direct read PDF file containing model; another is realized through software between the capture function.

2) The dynamic change of the position and orientation of the $3 \mathrm{D}$ model, or is the position of the observation point and orientation. This is one of the most basic interactive functions. Users can observe the $3 \mathrm{D}$ model from any direction, is not restricted.

3) Dynamic changes in the size of the 3D model. This is one of the most basic interactive functions. Users can accord their own needs to observe the local feature model.

4) Set the 3D scene background color. 3D graphics display in the scene, should be able to set the background for the scene. Like photography, we can choose what we want the background to shoot.

5) Display control object in the 3D scene. 3D scenes usually contain multiple geometric entities, we can control each geometric entity display: visible, invisible or transparent etc. In the complex 3D scene, we often need to be a part of the scene is not visible, or isolated in a part of the scene, and make the other part is not visible, so as to facilitate the observation.

6) Highlight selected objects. In some cases, we want to we selected in the $3 \mathrm{D}$ objects in the scene (perhaps by mouse click etc.) are highlighted to indicate indeed, chose the object, in this case, we need to have this function. This function is adapted to some specific interactive 3D applications.

7) Change the light effect. The real scene are visible with light effects, such as sunlight, moonlight, lighting, color light etc. We can change the light effect to simulate the real needs of our scene, let the objects displayed in what we hope for light environment. Players realize, for simplicity, only provides several special light to choose. More complex light environment can create using the same way.
8) Multi view observation. It is applied in some special occasions, such as the front view, top view and side view, back view etc.

9) The node tree and model of information acquisition. The node tree can view model of node level, select a node and the corresponding operation. Model of information can include model frame number, texture, as well as other want to obtain information and so on.

Therefore, the 3D geological mapping results show model integration process of platform design, we must solve two problems: 1) Model sharing problem, in order to reflect the integration goal, 2) Interface issues, design of man-machine interactive diversification model, reflect the display target.

\section{THE OVERALL DESIGN}

Combined with the platform needs can be informed, 3D geological mapping results of model integration platform, which comprises the following steps: (1) the import diversification model: take U3D as a general interface, realize the communication of geologic model and Acrobat platform in different software; (2) check the 3D model information in the Adobe Acrobat platform, the realization of inspection and adjustment of the model, and through the embedded Adobe 3D Reviewer editing tools make model re editing work; (3) increase the management and interaction in the Adobe Acrobat platform, realize the management and operation of the model; and using JavaScript script to achieve the advanced operation; system overall process as shown in figure 1:

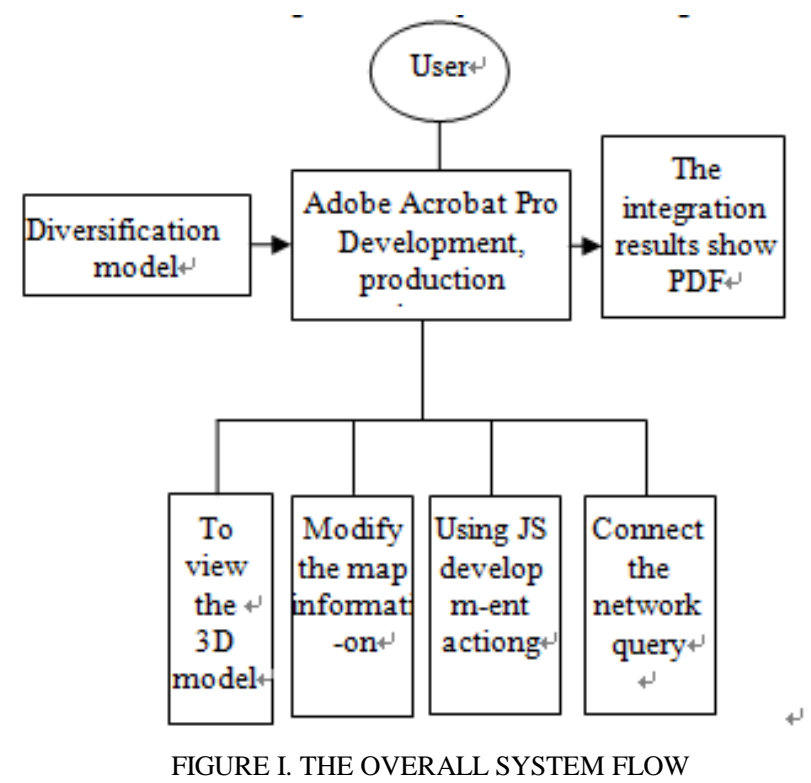

For different geologic model building software, model format differences software itself produces is great. In order to ensure the unity of all import Adobe model of Acrobat platform provides a general model to capture operation, process model captured as shown in figure 2 : 


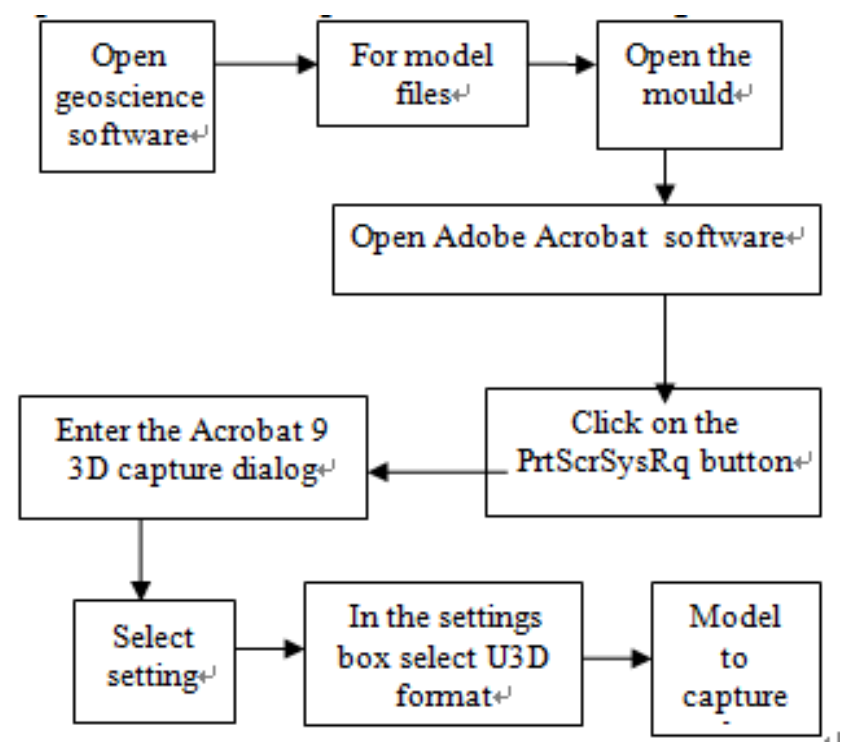

FIGURE II. MODEL CAPTURE PROCESS

\section{ANALYSIS AND IMPLEMENTATION OF KEY TECHNOLOGY OF INTERACTION}

In Adobe Acrobat, Javascript 3D is a hierarchical model. Operation from top to bottom, three-dimensional model of operation should be the first to get the 3D object, use getAnnot3D (nPage, cName) function to obtain the; in getAnnots3D, the nPage parameter contains the page number of the $3 \mathrm{D}$ object; the cName parameter indicates the name of the $3 \mathrm{D}$ object. And then through the context3D attribute function representation of the $3 \mathrm{D}$ scene, and then in the 3D scene through scene to obtain the specific model, and through the meshes attribute in the scene of each part of the model control and careful operation.

Now to refresh a geological model scene as an example, the code control processes are introduced:

context3D = getAnnots3D (0) [0].context3D;

context3D.makeAllMeshesInvisible();

this.resetForm();//(Start time (sequential) description, stimulate)

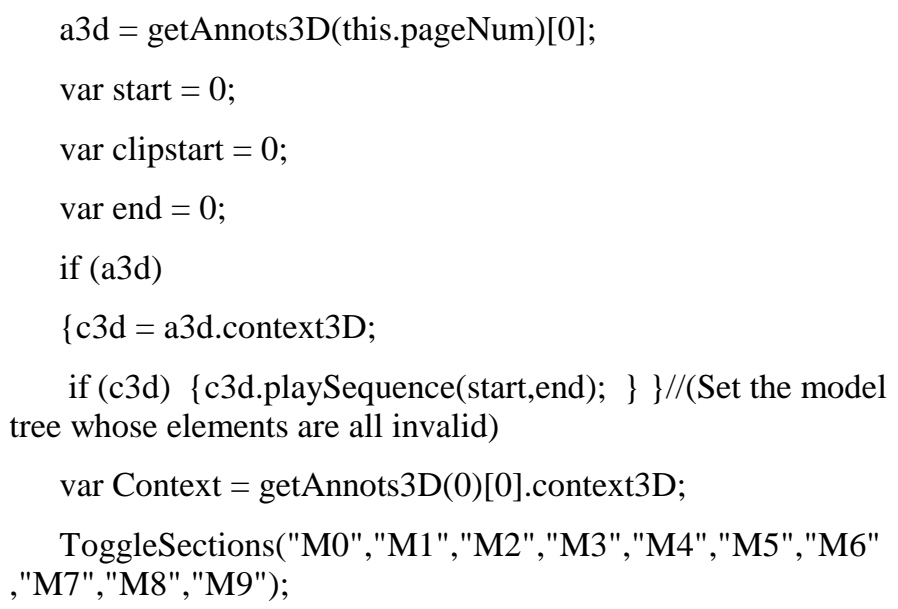

if (c3d) \{c3d.playSequence(start,end); \} $\} / /($ Set the model tree whose elements are all invalid)

var Context $=$ getAnnots3D $(0)[0] \cdot \operatorname{context} 3 \mathrm{D}$;

ToggleSections("M0","M1","M2","M3","M4","M5","M6" ,"M7","M8","M9");

function ToggleSections ()\{

for (var $\mathrm{i}=0 ; \mathrm{i}<$ ToggleSections.arguments.length;i++) \{

var mesh $=$

Context.scene.meshes.getByName(ToggleSections.arguments[i ]);

mesh.visible $=$ true; $\}$

\}//Make all the hidden attribute hidden control is invalid.

getField("Hide All").value = false

var Context=getAnnots3D(0)[0].context3D;

var $\mathrm{s}=$ Context.scene.meshes.getByName("M0");

if (s.visible==true) s.renderMode="solid";

In the PDF 3D platform, animation and video 3D model, can be added into various types, and can be used Javascript realize the diversification of control.

1)Show / hide an object in the scene, the key attribute set: visible, such as the scene of various objects called M12 display / hide

Var Context=getAnnots 3D(0)[0]. Context 3D;

Var S1=Context. Scene.meshes.getByName(“M12”);

S1.visible=true/false;

Note: If you need to display / hide the objects, the available cycle

for (var $\mathrm{i}=0 ; \mathrm{i}<$ ToggleSections.arguments.length;i++) \{

Var mesh =context.scene.meshes.getByName(ToggleSections.arguments [i]);

Mesh.visible=true/false; $\}$

2) Entity / transparent filling an object in the scene, the key attribute settings: renderMode. For example, in the scene of various objects called M0 entity / transparent filler

var Context=getAnnots3D(0)[0].context3D;

var $\mathrm{s}=$ Context.scene.meshes.getByName("M0");

if(s.visible==true) s.renderMode="solid"/"transparent";

$\mathrm{Or}$

var Contest=getAnnots3D(0)[0].context3D;

var s1=Contest.scene.meshes.getByName("M12");

s1.renderMode="solid"/"transparent";

3) Start / stop animation scene environment, the key attribute settings: restartAnimation (), setCameraMotion (). Such as the scene in the environment animation start / stop

getAnnots3D(0)[0].context3D.restartAnimation();

getAnnots3D $(0)[0]$.context3D.setCameraMotion (true/false); 
4) In the scene environment animation move in the opposite direction, the key attribute set: reverseAnimation (). As will be the scene of the environment animation move in the opposite direction

getAnnots3D(0)[0].context3D.reverseAnimation();

\section{CONCLUSION}

Three dimensional geological mapping results of model integration platform to display the main goal is to put the results of geological model, displayed, and by re decomposition, the observation model, understanding of model to help users better, it has great effect on the value and significance of non geological speciality or geological model of deep understanding of human cognition and understanding of geological mapping job.

At the same time, in the development process, found that Adobe Acrobat has two times the development of excellent function, wide application range, good containment, can include multiple file types such as Word, Excel, Flash, email, CAD file format, 3D file format, further study will contribute to more social scientific research achievements in the field of representation and expression, has practical application and popularization value.

\section{ACKNOWLEDGEMENT}

This research was financially supported by the China Geological Survey Foundation.No. 1212011220248.

\section{REFERENCES}

[1] Universal 3D (U3D) White Paper [S].http: / /www. 3di.forg .

[2] Adobe white papar.Composing PDF Documents with 3D Content from MicroStation, 2005

[3] Rick Benoit, Jeffery A. Williams.3D Industry Forum Announces Standard for 3D CAD Data Repurposing, 2004.

[4] Zhu Liangfeng. Analysis of Technology Research"Visualization of 3D geological model" [C]. The Thirteenth National Academic Conference on image and graphics, 2006.

[5] Qiao Baojuan,Liu Yan,Cai Guoqiang. PDF document generation technology and application in which PKPM statement [C], The Sixteenth National Engineering Design of computer application in the conference proceedings, 2012.

[6] Guo Qing,Wang Xiangyu,Wang Yuanzheng. U3D based development of aero engine turbine assembly simulator [J], Experimental technology and management, 2012 (4).

[7] Miao Yinghua,Su Honggen. The analysis of U3D file format and operation mechanism [J]. Computer applications and softwar, 2010 (02).

[8] Gong Weixue. Research and implementation of visualization techniques for geological body 3D geological mapping [D]. Jilin University, 2013.

[9] Ma Shilu,Xue Chengqi. Application of technology industry 3D PDF technology based on IETM, China manufacturing industry information $[\mathrm{J}], 2009$ (9) 